Philip van der Eijk

\title{
Gesundheit, Lebensstil und Verantwortung: Historische Wurzeln und gegenwärtige Perspektiven
}

\begin{abstract}
Health, Lifestyle and Responsibility: Historical Roots and Current Perspectives. The question to what extent health and disease are matters of individual and collective human responsibility was first raised and systematically discussed in ancient Greek medicine and philosophy in the $5^{\text {th }}$ and $4^{\text {th }}$ century BCE. This chapter discusses the consequences of these discussions for the definition of the aims and methods of the medical art, in particular the preservation and enhancement of health and the prevention of disease through lifestyle-related prophylactic and therapeutic measures. It also considers some of the implications of these ancient discussions for today's theory and practice of preventative and lifestyle-related medicine.
\end{abstract}

\section{Einleitung}

Im Januar 2015 publizierte der angesehene amerikanische Krebsforscher Bert Vogelstein von der Johns Hopkins University in Baltimore zusammen mit dem Mathematiker Cristian Tomasetti in der Zeitschrift Science einen vieldiskutierten Aufsatz über den sogenannten Pechfaktor bei der Entstehung von Krebserkran-

\begin{abstract}
Anmerkung: Die in diesem Aufsatz behandelte Thematik wurde in unterschiedlichen Versionen an der Berlin-Brandenburgischen Akademie der Wissenschaften, dem Berliner Medizinhistorischen Museum der Charité sowie an mehreren Universitäten und Akademien in Deutschland, den USA, China, Taiwan und Japan mündlich vorgetragen und hat von den Fragen und Anregungen der jeweiligen Zuhörer sehr profitiert. Der Aufsatz entwickelt Gedanken, die ansatzmäßig bereits zuvor skizziert wurden (van der Eijk 2011, 2013). Eine erweiterte Version, die ausführlicher die kulturhistorischen Rahmenbedingungen der beschriebenen Veränderungen im medizinischen Denken in der Antike berücksichtigt und auch das Verhältnis zwischen der Medizin und dem Heilkult des Asklepios behandelt, erscheint demnächst in englischer und chinesischer Sprache im Museum Sinicum (van der Eijk 2021). Der Autor dankt dem Einstein Center Chronoi für die finanzielle Unterstützung der Forschungsarbeit, aus der dieser Aufsatz hervorgegangen ist.
\end{abstract}

Philip van der Eijk, Interdisziplinäre Arbeitsgruppe „Zukunft der Medizin: Gesundheit für alle“, Berlin-Brandenburgische Akademie der Wissenschaften; Institut für Klassische Philologie, Humboldt-Universität zu Berlin

Ә OpenAccess. ( 2021 Philip van der Eijk, publiziert von De Gruyter. (cc) BY-NC-SA Dieses Werk ist lizenziert unter einer Creative Commons Namensnennung - Nicht kommerziell - Weitergabe unter gleichen Bedingungen 4.0 International Lizenz. https://doi.org/10.1515/9783110713336-004 
kungen (Tomasetti \& Vogelstein 2015). Vogelstein und Tomasetti schätzten, dass das lebenslange Risiko für die Entwicklung eines Tumors zu ungefähr 65\% auf Fehlern der DNA beruht, die zufällig entstehen und zu Krebs führen können.

Diese Publikation rief in der Öffentlichkeit einen Sturm von Reaktionen hervor. In den Schlagzeilen der Zeitungen hieß es, wissenschaftlich sei jetzt nachgewiesen, dass es hauptsächlich eine Frage von Pech oder Glück sei, ob man eine Krebserkrankung entwickelt oder nicht. Es sei also egal, ob man gesund oder ungesund lebt, ob man regelmäßig zur ärztlichen Vorsorgeuntersuchung geht oder nicht, kurz gesagt: Es sei also unabhängig davon, wie verantwortlich oder unverantwortlich man mit seiner Gesundheit umgeht.

Ein Jahr später aber haben Tumorbiologen der Stony Brook University New York Vogelsteins Hypothese weitgehend korrigiert: Durch eine neue statistische Auswertung derselben Daten gelangten sie zu der Schlussfolgerung, dass tatsächlich weniger als ein Drittel der Krebsfälle dieser Art von Pech zuzuschreiben ist und dass eine gesunde Lebensführung und Umwelt durchaus die Wahrscheinlichkeit von Krebs erheblich reduzieren (Wu et al. 2015).

Wie es möglich sein kann, dass wissenschaftliche Untersuchungen identischer Datensätze zu derart unterschiedlichen Ergebnissen kommen, ist an sich eine interessante Frage - und tatsächlich ist die Debatte um Vogelstein und Tomasettis Bad Luck Theory noch weitergegangen. Mir geht es in dem vorliegenden Aufsatz aber um die etwas allgemeinere Frage, inwieweit Gesundheit und Krankheit - und das gilt nicht nur für Krebserkrankungen, sondern für sehr viele Krankheiten - eine Frage von Glück oder Pech sind und dem Zufall - manche wurden sagen: dem Schicksal - zuzuschreiben sind oder ob sie auf Faktoren zurückgehen, die grundsätzlich innerhalb menschlicher Kontrolle liegen. Anders gesagt: Bis zu welchem Ausmaß sind Gesundheit und Lebensqualität ,machbar‘, d. h. kontrollierbar? Welches Potenzial für die Eigenleistung des Menschen bzw. des potenziellen Patienten gibt es hier und wie kann man dazu beitragen, dass dieses Potenzial optimal genutzt wird? Etwas schärfer und provokanter formuliert: Inwieweit sind wir für unsere Gesundheit verantwortlich - sowohl individuell als auch als Kollektiv - und für die Gesundheit unserer Kinder oder die Gesundheit anderer Mitglieder unserer Gesellschaft? Diese Fragen sind wesentlich für unser Nachdenken über Gesundheit und Lebensqualität, Krankheitsvorbeugung und Altersvorsorge, aber auch für die Gesundheitspolitik, für die Organisation des Gesundheitssystems und für Krankenversicherungen. Man muss nur an die riesigen Gesundheitsprobleme denken, die es in unserer heutigen Gesellschaft wegen der sogenannten verhaltensbedingten Erkrankungen (lifestyle related illnesses) zu bewältigen gibt, wie etwa gewisse Formen von Adipositas oder Diabetes mellitus. 
Die Reflexion dieser Fragen ist nicht neu, sie hat vielmehr eine lange Geschichte. In diesem Aufsatz möchte ich zu den Wurzeln dieses Denkens zurückgehen, ja zu den Wurzeln unserer medizinischen Wissenschaft, nämlich zum antiken Denken über Gesundheit von Leib und Seele. Denn die grundsätzliche Frage nach der Machbarkeit von Gesundheit und der Mitverantwortlichkeit für Gesundheit wurde zum ersten Mal im griechisch-römischen Altertum gestellt: von Ärzten wie Hippokrates von Kos (460 - 370 v. Chr.), Diokles von Karystos (4. Jh. v.Chr.) und Galen von Pergamon (129-216 n.Chr.), und von Philosophen wie Aristoteles (384-322 v. Chr.) und Platon (428-348 v. Chr.). Zusammen bildeten sie die Grundlagen der medizinischen Wissenschaft, wie wir sie heute kennen: Sie haben die Entwicklung der westlichen Medizin ganz entscheidend geprägt.

\section{Das Gesundheitsverständnis der antiken Medizin}

Die antiken griechischen Ärzte und Philosophen haben zu ihrer Zeit große Veränderungen im Denken über Gesundheit ausgelöst. Denn sie wehrten sich gegen eine ältere Auffassung, die vor ihrer Zeit, im archaischen griechischen moralischen Denken (der Zeit von Homer, um 700 v.Chr.), aber auch im antiken Mesopotamien und Ägypten weit verbreitet war. Nach dieser älteren Auffassung ist Gesundheit eines der sogenannten ,äußeren Güter‘, also vergleichbar mit adliger Herkunft, vererbtem Reichtum, Ansehen und körperlicher Kraft und Schönheit. Gesundheit wird hier als ein von der Natur gegebenes Gut betrachtet, das man von Geburt oder zufällig, vielleicht auch von den Göttern geschenkt bekommen hat, das man aber einfach zu akzeptieren hat und worüber man selbst keinerlei Kontrolle hat.

Gegen diese Auffassung wehrte sich die antike medizinische Forschung, die sich im Laufe des 5. Jahrhunderts v. Chr. entwickelte und deren Anfänge meist mit dem Namen des Hippokrates von Kos verbunden werden (obwohl es in Wirklichkeit um eine größere Gruppe von Ärzten ging, die nicht notwendig alle Schüler des Hippokrates gewesen sind, sondern auch selbständig gearbeitet haben). ${ }^{1}$ Die hier aufkeimende medizinische Forschung suchte die Ursachen von Gesundheit und Krankheit im menschlichen Körper selbst, in der Natur und im Verhältnis

1 Für eine Gesamtdarstellung der medizinischen Ansichten und Praktiken, die in der Sammlung der Schriften, die unter dem Namen des Hippokrates überliefert ist, belegt sind: Flashar 2016. Zur Frage nach dem Verhältnis zwischen diesen Schriften und dem historischen Hippokrates: van der Eijk 2016. 
zwischen Menschen und Umwelt. Der Begriff ,Gesundheit‘ gewann an Bedeutung; und das Wort hygieia (ن́yícı), das bisher nur sehr selten in griechischen Texten auftauchte - und dann vorwiegend im Sinne von körperlicher Kraft - nahm in den medizinischen Texten eine zentrale Bedeutung an. Die antike Medizin bemühte sich aktiv darum, die Gesundheit durch eine bestimmte Lebensführung (vor allem durch Ernährung und Bewegung) und mit natürlichen und menschlichen Mitteln zu fördern, Krankheiten vorzubeugen und eine möglichst hohe Lebensqualität zu schaffen. Sie entwickelte den Gedanken, dass der Mensch seine eigene Gesundheit - und zugleich die der sozialen Verbände, in denen er lebt - bis zu einem erheblichen Grad mitbestimmen kann und dafür auch Verantwortung zu übernehmen hat.

Die Gesundheit wurde somit als ein grundsätzlich erreichbarer Zustand betrachtet, als etwas, wofür man sich selbst einsetzen kann, wofür man sich auch anstrengen kann und worum man kämpfen muss, wenn nötig mit Hilfe eines Experten, eines Arztes.

In dieser Vorstellung ging es nicht einfach darum, von Krankheit geheilt $\mathrm{zu}$ werden: Aufgabe der Medizin, d.h. Aufgabe des Arztes und des Patienten, war es nach den antiken Vorstellungen auch, Krankheit durch eine gesunde Lebensführung vorzubeugen. Aufgabe der Medizin war es zudem, Hilfe zu bieten, wenn Menschen an einer unheilbaren chronischen Erkrankung leiden und ihnen trotz Beschränkungen und Behinderungen eine möglichst hohe Lebensqualität zu gewährleisten: dies äußerte sich in dem berühmten hippokratischen Kernsatz „helfen, oder (zumindest) nicht schaden“ (van der Eijk 2005, S. 101-118). ${ }^{2}$

So entwickelte sich in der griechischen Medizin der Gedanke, dass es für jeden Menschen wichtig ist, bewusst und verantwortlich mit dem eigenen Körper umzugehen, mit seinen Möglichkeiten und seiner Gebrechlichkeit, mit seinem Potenzial zum Genießen und mit seiner Empfindlichkeit und Verletzbarkeit. Zu dieser Mitverantwortlichkeit für den Körper gehörte eben auch, dass man medizinische Hilfe sucht, wenn man sie braucht. ${ }^{3}$ Zum einen, indem man Krankheiten, von denen man meint, dass sie durch gewisse Angewohnheiten verursacht wer-

2 Die unter dem Namen von Hippokrates überlieferten medizinischen Schriften sind in Bezug auf diesen Punkt nicht gleichlautend. Die dominierende Tendenz ist aber, dass der Arzt auch in Fällen von unheilbarer Erkrankung helfen soll. Die Auffassung, dass man Patienten mit unheilbarer Krankheit am besten nicht behandelt - die sogenannte ,Unterlassung medizinischer Hilfeleis-

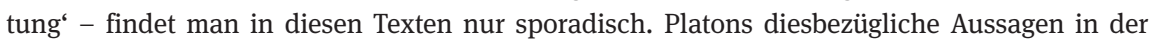
Politeia betreffen ganz spezifisch die übertriebenen diätetischen Behandlungsmethoden eines gewissen Herodikos, nicht aber die Medizin, die er mit Asklepios assoziiert.

$3 \mathrm{Zu}$ dieser, quest for health', und zum Asklepioskult als eine alternative Antwort auf diese Suche: van der Eijk 2021). 
den, durch eine gesunde Lebensführung und medizinische Beratung vorzubeugen versucht. Zum anderen, indem man durch eine gesunde Lebensführung den Körper widerstandsfähig (resilient) macht und auf Situationen vorbereitet, in denen man eine größere medizinische Behandlung überstehen muss. Zum dritten gehörte dazu auch, dass man im Fall einer bereits eingetretenen Krankheit aktiv nach Heilung sucht und an der Behandlung mitwirkt. Der Wille und die Motivation des Patienten, geheilt zu werden, galten als entscheidende Voraussetzungen. Das ist gar nicht so selbstverständlich, wie es zunächst scheint. Heilung setzt Anstrengung und Kampf voraus: einen Kampf gegen die Macht der Erkrankung, gegen die Schwäche des Willens und gegen Unglauben und Verzweiflung des Patienten. In einem antiken medizinischen Text heißt es, dass Arzt und Patient gemeinsam der Krankheit widerstehen müssen und dass sich beide in einem Kampf befinden, der mit Anstrengung und Mühe einhergeht (Hippokrates, „Epidemien“, I.11, Müri 1986 [1938], S. 10 -11).

Dies sind die zentralen Grundsätze des antiken medizinischen Denkens, wie es im 5. und 4. Jahrhundert v.Chr. von Hippokrates und von anderen Ärzten entwickelt wurde. Um sie besser zu verstehen, sei auf zwei weitere wichtige Komponenten hingewiesen.

Die erste ist die Ätiologie, das Interesse an Ursachenforschung. Wie gesagt suchte die antike medizinische Forschung die Ursachen von Gesundheit und Krankheit im menschlichen Körper selbst, in der natürlichen Welt und im Verhältnis zwischen Menschen und Umwelt. Dazu entwickelte sie einen äußerst raffinierten Kausalitätsbegriff, in dem unterschiedliche Arten der Verursachung bestimmt wurden: innere und äußere Ursache, Hauptursache und Nebenursache, Voraussetzung, Ermöglichung, conditio sine qua non, vorhergehende Ursache, strukturelle Ursache, Begleiterscheinung, Symptom, Katalysator, Hauptwirkung, Nebenwirkung usw. ${ }^{4}$ Dieses Interesse an Ursachen hatte die griechische Medizin gemeinsam mit anderen Bereichen der antiken Gesellschaft, wie etwa der Philosophie, der Naturwissenschaft, der Geschichtsschreibung und auch dem Recht. Aitia (aítía), das Wort, das wir mit Ursache oder Grund übersetzen, bedeutet ja zugleich Schuld, Verantwortlichkeit, Zurechnung; und um die Bestimmung von Schuld und Verantwortung bemühten sich ja auch die Anwälte in den athenischen Gerichtshöfen. Auch dort, im griechischen Rechtsdenken und Gerichtsverfahren, gab es ein differenziertes Verständnis von verschiedenen Arten der Verantwortung, Schuld und Zurechnung. Viele dieser Unterscheidungen im Bereich der Kausalität haben von ihrer Gültigkeit in der heutigen Medizin - wie auch im heutigen Recht - nichts verloren. Was aber einen wesentlichen Unterschied

4 Für eine Darstellung antiker medizinischer Auffassungen zur Kausalität: Hankinson 2001. 
zwischen dem heutigen und dem antiken Denken ausmacht, ist der quantitative Ansatz: Nämlich das Messen des relativen Gewichts eines bestimmten kausalen Faktors und die Bestimmung der relativen Wahrscheinlichkeit einer gewissen Erkrankung bzw. die Bestimmung der Erfolgsquote eines gewissen Heilmittels anhand statistischer, aufgrund von empirischen bzw. experimentellen Untersuchungen in einer umfangreichen Population gewonnener Daten. Große quantitative epidemiologische Untersuchungen waren in der Antike aus praktischen Gründen nicht durchführbar. Ein gewisses Bewusstsein von Allgemeinheit, Häufigkeit, Regelmaß, Seltenheit und Ausnahme war aber durchaus vorhanden.

Der zweite wichtige Aspekt antiker Medizin ist die Diätetik, die Lehre des

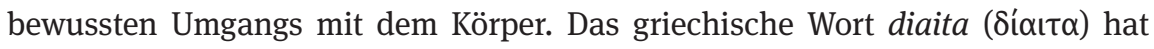
dabei ein viel breiteres Bedeutungsspektrum als unser Begriff ,Diät‘. Diaita umfasst nicht nur gesundes Essen und Trinken, sondern auch Arbeit, Erholung, Sport, Massage, Stimmübung, Baden, Körperpflege, Schlafen und Wachen, Sexualität sowie dasjenige, was wir heutzutage als Fitness und Wellness bezeichnen. ${ }^{5}$ Neben Chirurgie und Pharmakologie galt die Diätetik als einer der drei wichtigen Teilbereiche der Medizin; sie war aber nicht nur eine Methode zur Behandlung von Krankheiten, sondern auch einer der wichtigsten Bestandteile der Gesundheitsvorsorge. Es ging hier um eine strukturierte Lebensführung, die von den griechischen und römischen Ärzten nicht nur mündlich vermittelt, sondern auch schriftlich verbreitet wurde. Dies hatte zum Ziel, möglichst viele Menschen dazu zu befähigen, ihre eigene Gesundheit - und die ihrer Kinder und anderer Verwandter - zu fördern und Krankheiten vorzubeugen, ohne immer von der Anwesenheit und der Kompetenz eines Arztes abhängig zu sein. Die Lebensregeln variierten je nach Geschlecht, Alter, sozialer und wirtschaftlicher Lage, Klima und Lebensumständen der Person, für die sie gemeint waren. Aus diesem Grund verfassten die griechischen medizinischen Schriftsteller nicht nur wissenschaftliche Abhandlungen, sondern auch popularisierende Texte, die für Laien zugänglich waren. Es ging darum, das für jeden einzelnen Menschen passende, je nach persönlichen Umständen unterschiedlich gestaltete Gleichgewicht zu finden. Diokles von Karystos, ein Arzt und medizinischer Autor aus dem 4. Jahrhundert v.Chr., verweist in seinen diätetischen und therapeutischen Schriften auf ,das Passende“ (to harmotton), das für jeden Menschen unterschiedlich sein kann. Folglich finden sich in seinen Schriften und in denjenigen seiner Zeitgenossen Lebensregeln für Reisende, für Seefahrende, für Jüngere und Ältere und für Ledige und Arbeitsame, sowie Vorschriften über die beste Zube-

5 Für eine ausführliche Darstellung (in deutscher Sprache) der Theorie und Methode der antiken Diätetik und der praktischen Durchführung ihrer Maßnahmen: Wöhrle 1990. 
reitung von Speisen und das richtige Verhalten beim Weintrinken während des Symposions.

$\mathrm{Zu}$ den von den griechischen Ärzten in diesem Zusammenhang behandelten Themen gehörten auch Kindererziehung und die Versorgung älterer Menschen. So widmete Galen von Pergamon ein ganzes Buch seiner Schrift über die Gesundheit der Frage, wie man auch in hohem Alter das Leben genießen und sein Potenzial als Mensch zur Entfaltung bringen kann. Ziel der Diätetik war also nicht nur die Vorbeugung von Krankheiten, sondern auch die Erhöhung der Gesundheit und der Lebensqualität entsprechend den Möglichkeiten, die die Situation des jeweiligen Menschen bot - auch in Zuständen von Behinderung oder unheilbarer chronischer Erkrankung. Denn Gesundheit und Lebensqualität, so führte Galen aus, sind relative, graduelle Dinge, die für jeden Menschen in jeder Lebensphase unterschiedlich gestaltet sein können: in Jugend und Alter, in Zuständen körperlicher Fitness, aber auch in Zuständen von Schwäche, abnehmender Kraft und chronischer Behinderung. ${ }^{6}$

Diese antiken Gedanken sind von erstaunlicher Aktualität. Denn noch heutzutage lesen wir in den Zeitungen, in den Magazinen der Krankenkassen und auf den Flyern im Wartezimmer des Arztes über die Wichtigkeit einer gesunden Lebensführung, z.B. zur Vorbeugung von Krankheiten wie Diabetes oder Bluthochdruck und zur Bewältigung von Stress. Auch die Frage, wie man trotz Beschwerden, Behinderung oder unheilbarer chronischer Krankheit eine angemessene Lebensqualität erreichen kann, findet in der heutigen Medizin und Gesellschaft viel Beachtung. Ein dritter Punkt, der heutzutage (wieder) viel diskutiert wird, ist die personalisierte oder individualisierte Medizin, die Bestimmung eines therapeutischen Trajektes, das ganz präzise auf den individuellen Gesundheitszustand eines Menschen abgestimmt ist: Auch dieser Gedanke wurde bereits in der antiken Medizin, nämlich in ihrer Betonung des für jeden Menschen Angemessenen, eingeführt. Galen spricht in diesem Zusammenhang von der ,Eigentümlichkeit' des individuellen Gesundheitszustandes des einzelnen Menschen: So viel die Patienten, die an derselben Erkrankung leiden, auch gemeinsam haben, so hat jeder Mensch, jeder Patient, dann doch seine oder ihre eigene Beschaffenheit; und es kommt darauf an, diesen Besonderheiten in der medizinischen Versorgung dann Rechnung zu tragen. In einer der Hippokrates zugeschrieben Schriften, den sogenannten „Epidemien“ (Buch 1, Kap. 23) heißt es, dass der Arzt sowohl die gemeinsame Natur aller Menschen als auch die eigen-

6 Für eine allgemeine Übersicht von Galen von Pergamon: Schlange-Schöningen 2003; zu seinen Auffassungen zur Diätetik: Wöhrle 1990. 
tümliche Natur eines einzelnen Menschen berücksichtigen muss, um die richtige Diagnose zu erstellen und die angemessene Behandlung zu bestimmen.

\section{Gesundheit der Seele}

Unser nächstes Thema ist die Gesundheit der Seele, und diese bringt uns zu den Philosophen, insbesondere zu Aristoteles. Denn auch in der griechischen Philosophie gewann die Gesundheit an Bedeutung. So billigte Aristoteles in seiner Ethik der körperlichen Gesundheit eine wichtige Rolle für moralisches und intelligentes Handeln zu. Er war der Ansicht, dass geistige und körperliche Gesundheit eng miteinander verbunden sind: Zur Ausübung der charakteristischen menschlichen seelischen Fähigkeiten - Vernunft, Sprache, moralische Überlegung - für sittliches und gesellschaftliches Leben und Handeln bedarf es einer gesunden körperlichen Grundlage, während umgekehrt ein ungesunder geistiger Zustand (etwa Stress, Depressivität oder Obsession) negativ auf den Körper einwirken kann. ${ }^{7}$ Auch in diesem Bereich der geistigen Gesundheit und ihrer Wechselwirkung mit dem Körper sieht Aristoteles eine entscheidende Rolle für die menschliche Verantwortung. Die Gesundheit gilt hier wiederum als ein erreichbarer Wert, als etwas, das zumindest bis $\mathrm{zu}$ einem gewissen Grad im Bereich menschlicher Kontrolle liegt. Gesundheit ist also nicht länger eines der ,äußeren Güter', die man als Schicksalssache hat oder nicht hat (Glück oder Pech). Und die Gesundheit wird nicht schlechthin und negativ als ,Abwesenheit von Krankheit“ definiert, sondern sie wird als positiver, produktiver Zustand verstanden, den es $\mathrm{zu}$ entwickeln und $\mathrm{zu}$ optimieren gilt. ${ }^{8}$ Ziel ist, sich von Anfang an um eine gesunde Lebensführung in beiderlei Hinsicht zu bemühen (Körper und Geist). Aristoteles führt hier aus: Man muss sich um ein inneres Gleichgewicht zwischen Seele und Körper kümmern, denn wenn man dieses Gleichgewicht verliert, läuft man die Gefahr, in einen dauerhaften Zustand von Instabilität zu geraten, den man nicht mehr kontrollieren oder bessern kann und der zu moralischem Missverhalten und sogar zu zwanghaften Störungen führen kann.

Fast das gesamte siebte Buch seiner Nikomachischen Ethik widmet Aristoteles dem Problem der Willensschwäche, der akrasia ( $\alpha \kappa_{\alpha} \alpha \sigma^{\prime} \alpha$ ), einer Art krankhafter Mangel an Selbstkontrolle, die den Menschen in seinen moralischen Entscheidungen entgleisen lässt. Akrasia bedeutet, dass man Dinge tut, von denen man

7 Für eine ausführlichere Darstellung von Aristoteles` Ansichten zum Verhältnis zwischen Körper und Seele und den Konsequenzen für die hier besprochene Thematik mit Hinweisen auf einzelne Stellen und die einschlägige Sekundärliteratur: van der Eijk 2000

8 Zum Gesundheitsverständnis des Aristoteles: Tracy 1969. 
weiß, dass sie schlecht für einen sind; und wenn man sie getan hat, bedauert man die Handlung und hat ein schlechtes Gewissen; man möchte gerne anders handeln, aber man hat nicht die Kraft, sich für das Gute zu entscheiden, der Wille ist nicht stark genug. Man könnte darin eine antike Parallele zu unserem heutigen Begriff der Sucht sehen. Aristoteles kommt auf dieses Thema zu sprechen, weil er daran interessiert ist, in welchem Ausmaß moralisches Fehlverhalten zu entschuldigen ist, falls ein gewisser krankhafter Zustand vorherrscht, der das Urteilsvermögen und die Entscheidungsfähigkeit eines Menschen beeinflusst. Der Kontext ist also wiederum ein moralischer, sogar ein juristischer: Kann man einem Menschen, der in einem solchen Zustand eines gestörten seelischen Gleichgewichts handelt, seine Handlungen zurechnen? Aristoteles untersucht hier die Grenzen zwischen dem Moralischen und dem Klinischen, dem Ethischen und dem Pathologischen. In diesem Zusammenhang ist für Aristoteles entscheidend, was die Ursache eines solchen geistigen Zustandes ist. Anders gesagt: Wie hat es so weit kommen können? Ein Mensch, der in einem Zustand von Trunkenheit einen Unfall verursacht und einen anderen Menschen verletzt, ist sich vielleicht nicht darüber im Klaren, was er tut, aber er ist trotzdem schuldig, weil es in seiner Kontrolle lag, den Zustand der Trunkenheit zu vermeiden. Aristoteles vergleicht dies mit dem Werfen eines Steines: Wenn man den Stein einmal in Bewegung gebracht hat, kann man ihn nicht länger in seiner Bewegung beeinflussen oder zum Stillstand bringen; aber die Entscheidung, den Stein zu werfen oder ihn nicht zu werfen, ist durchaus etwas, das innerhalb unserer Kontrolle liegt (Aristoteles 2006, Nikomachische Ethik, 1114a17). Ein ungesunder Zustand der Seele ist also nicht automatisch eine Entschuldigung für Fehlverhalten: Man muss zuerst bestimmen, wie die Seele in einen solchen ungesunden Zustand geraten ist und wer dafür verantwortlich ist. Die Willensschwäche ist nicht einfach von Natur aus da, sie ist nicht etwas Unvermeidliches, das mit unserer genetischen Veranlagung gegeben ist, sondern sie ist das Resultat eines gewissen Lebensstils. Die Wahl dieses Lebensstils wiederum wurde getroffen, als man geistig fähig war, als man noch gesund war und bevor man in einem Zustand geriet, in dem man nicht länger in einer verantwortlichen Art und Weise urteilen und handeln kann (Nikomachische Ethik, 1114a21-31). Die Lösung liegt nach Aristotles in einer geistigen diaita, einer strukturierten seelischen Lebensführung, durch die man versuchen muss, auf dem richtigen Weg zu bleiben und sein inneres Gleichgewicht und somit seine Entscheidungsfähigkeit zu behalten. Und wenn es dann einmal schiefgeht, soll man professionelle Hilfe suchen - bei einem Arzt, einem Therapeuten, oder einem Berater, vielleicht einen Philosophen oder einen religiösen Seelsorger - und aktiv an der Behandlung mitwirken. Aristoteles beobachtet aber auch, dass viele Patienten nicht auf ihre Therapeuten hören, weil sie keine Lust haben, ihren Lebensstil zu ändern (Nikomachische Ethik, 1114a15-16). Das Pro- 
blem der ,patient compliance' war auch in der Antike schon bekannt. Sowohl präventiv (in der Instandhaltung und Optimierung der geistigen Gesundheit), als auch therapeutisch (in der Korrektur eines ungesunden seelischen Zustandes), gibt es nach Aristoteles also eine Rolle für die menschliche Verantwortung, sowohl individuell als auch kollektiv auf gemeinschaftlicher Ebene. Man muss die Patienten erziehen, und dafür braucht man ein gutes Bildungssystem und ein gesellschaftliches Gesundheitssystem, das die Gesundheitsversorgung gewährleisten sollte.

\section{Gegenwärtige und zukünftige Perspektiven}

Bisher haben wir vor allem darüber gesprochen, was die antiken Ärzte und Philosophen über die menschliche Verantwortung in Fragen der Gesundheit und Krankheit zu sagen hatten. Natürlich war die antike Medizin sich auch darüber bewusst, dass es Grenzen gibt an dem, was der Mensch und die Medizin leisten kann: Viele Krankheiten werden von Faktoren verursacht, auf die der Mensch keinen Einfluss hat oder die noch nicht geklärt sind; und viele Krankheiten sind (noch) unheilbar, gegen sie ist auch die professionelle Medizin ratlos. Das ist auch heute, trotz aller Fortschritte, immer wieder der Fall, wie die COVID-19-Pandemie uns allen deutlich gemacht hat. Auch diese Krise ruft übrigens reichlich Fragen nach Verhalten und Verantwortung auf, sowohl was das Entstehen und die Verbreitung der Erkrankung betrifft, als auch im Hinblick auf die Versuche zur Bewältigung der Pandemie auf individueller, (gesundheits-)politischer, gesellschaftlicher und wirtschaftlicher Ebene.

Das bringt uns zur Gegenwart und zur Zukunft der Medizin. Denn auch in der heutigen Gesellschaft ist wieder häufig von der Wichtigkeit der Vorbeugung die Rede: Medizin ist nicht nur kurativ, sie soll auch präventiv sein. Wie wir gesehen haben, ist dieser Gedanke nicht neu, er ist schon mehr als zweitausend Jahre alt. Das wirft aber die Frage auf, warum es immer noch oder immer wieder so schwierig ist, diese vernünftigen Prinzipien in der Realität der medizinischen Versorgung und Praxis umzusetzen.

Einige Ursachen haben wir schon gesehen: Prävention erfordert die Mithilfe der (potenziellen) Patientinnen und Patienten, und diese haben nicht immer die richtigen Kenntnisse, die nötige Ausbildung oder die erforderlichen materiellen Verhältnisse, um sich verantwortungsvoll um ihre Gesundheit und deren Erhaltung zu kümmern: sie brauchen Beratung, Unterstützung und intensive Betreuung und das macht die Implementierung präventiver Maßnahmen schwierig und aufwendig. Manchmal sind Menschen auch nicht wirklich willens, ihr Verhalten zu ändern und schlechte Gewohnheiten aufzugeben - das haben wir schon bei 
Aristoteles gesehen - oder sie befinden sich in einem sozialen Umfeld, das nicht gerade förderlich ist: Wer in problematischen Verhältnissen aufgewachsen ist, hat es viel schwieriger, sein Verhalten zu ändern und einen gesunden Lebensstil zu entwickeln und langfristig beizubehalten.

Es liegt aber nicht nur an den Patientinnen und Patienten selbst oder an ihren sozialen Verhältnissen. Auch in der medizinischen Forschung, Ausbildung und Praxis hat die Lebensstilmedizin es schwierig, sich durchzusetzen, es gibt gleich mehrere Hürden (Korteweg 2020). Manche dieser Hürden sind ideologischer Art und haben mit einer gewissen Mentalität zu tun, oder sie sind auf gewisse Traditionen und Gewohnheiten zurückzuführen. So ist die Effektivität der Lebensstilmedizin nur sehr schwer wissenschaftlich und experimentell nachweisbar obwohl es zu Diabetes Typ 2 jetzt überzeugende Untersuchungen gibt (Molema et al. 2019). Manche Hürden sind wirtschaftlicher, finanzieller oder praktischer Art und haben mit Problemen bei der Abrechnung gewisser lebensstilbezogener ärztlicher Leistungen oder mit ihrer versicherungstechnischen Anerkennung zu tun - obwohl es auch hier in den letzten Jahren große Verbesserungen gab: viele Krankenkassen in Deutschland vergüten regelmäßige Fürsorgeuntersuchungen und auch in den Niederlanden ist eine ,ganzheitliche Lebensstilentwicklung (,gecombineerde leefstijlinterventie') fester Bestandteil des Versicherungspakets. Aber auch der Zeitdruck, unter dem das medizinische Personal steht, spielt eine Rolle: Manchmal ist es einfacher und schneller, ein Medikament zu verschreiben als eine zeitaufwendige Verhaltenstherapie mit Lebensstilberatung und Betreuung durchzuführen.

Derartige Probleme sind grundsätzlich lösbar, dies würde aber Veränderungen im Gesundheitssystem erforderlich machen, die womöglich den wirtschaftlichen Interessen einiger Stakeholder zuwiderliefen. Ironischerweise sollten aber gerade ökonomische Argumente hier großes Gewicht haben: In der Diskussion über die Bezahlbarkeit der Gesundheitsversorgung wird immer wieder darauf hingewiesen, dass die Prävention von Krankheiten auf Dauer viel kostengünstiger und effektiver ist. In diesem Komplex zahlreicher Faktoren und unterschiedlicher Interessen sollte eine ausgewogene, am Gemeinwohl ausgerichtete Gesundheitspolitik das Ruder übernehmen.

Wichtig ist darüber hinaus ein guter Umgang mit Fragen von Verantwortung und Schuld. Offensichtlich ist es einseitig und kontraproduktiv, die Schuld für eine ungesunde Lebensführung nur beim Patienten zu sehen (victim blaming), denn immer gibt es neben der individuellen auch eine kollektive, gesellschaftliche Verantwortung. Aber sogar dann, wenn man den Begriff Schuld vermeiden möchte und lieber von Verantwortung oder noch positiver von einer Optimierung des individuellen Gesundheitspotenzials redet, bleibt die Frage, wem gegenüber ein Mensch eine solche Verantwortung für seine Gesundheit hätte bzw. für wen er 
sein Gesundheitspotenzial denn optimieren soll: Für die Familie, die mit den Konsequenzen leben müsste? Für die Gesellschaft, die die Kosten der Pflege tragen müsste? Wie man diese Fragen auch beantworten möchte, so ist es auf jeden Fall klar, dass man von einer moralischen oder gar gesetzlichen Pflicht zur Gesundheit nicht sprechen sollte - zumindest nicht, solange man die Gesundheit anderer Menschen nicht gefährdet (Stichwort Impfpflicht gegen COVID-19). Ein Arzt kann einen Patienten ja nicht dazu verpflichten oder zwingen, ein Medikament zu nehmen; es muss freiwillig bleiben. Aber gerade vor diesem Hintergrund ist es umso wichtiger und im Interesse aller, Menschen $\mathrm{zu}$ überzeugen und sie dazu anzuregen und zu ermutigen, an ihrer eigenen Gesundheit zu arbeiten, sie dabei gut zu informieren (siehe ,Citizen Science') und mit Beratung und Betreuung zu unterstützen.

Die Möglichkeiten und die Grenzen der menschlichen Verantwortung in Fragen der Gesundheit und Krankheit sind ein wichtiges Thema, zu dessen Erforschung die antike Medizin entscheidende Anstöße gegeben hat. Schon die antike Medizin forderte uns alle dazu auf, konstruktiv darüber nachzudenken, was man selbst - alleine oder mit professioneller Hilfe - zu seiner eigenen Gesundheit und der seiner Mitmenschen beitragen kann. Dieser Anspruch hat in zweitausend Jahren nichts von seiner Gültigkeit verloren.

\section{Literatur}

Aristoteles (2006): Nikomachische Ethik (Ursula Wolf, Übers.). Reinbek bei Hamburg: Rowohlt Taschenbuch.

Flashar, Hellmut (2016): Hippokrates. Meister der Heilkunst. Leben und Werk. München: C.H. Beck.

Hankinson, Robert J. (2001): Cause and Explanation in Ancient Greek Thought. Oxford: Oxford University Press.

Korteweg, Niki (2020): „Ontzieken: Gezond gaan leven als medicijn“. https://www.nrc.nl/ nieuws/2020/01/03/ontzieken-gezond-gaan-leven-als-medicijn-a3985651, besucht am 4.1.2020.

Molema, Hanneke/van Erk, Marjan/van Winkelhof, Martijn et al. (2019): Wetenschappelijk bewijs leefstijlgeneeskunde. Den Haag: Nederlands Innovatiecentrum voor leefstijlgeneeskunde (Lifestyle4health), http://bit.ly/ wetenschappelijkbewijsleefstijlgeneeskunde, besucht am 11.11.2020.

Müri, Walter (1986 [1938]): Der Arzt im Altertum. Darmstadt: Wissenschaftliche Buchgesellschaft.

Schlange-Schöningen, Heinrich (2003): Die römische Gesellschaft bei Galen. Biographie und Sozialgeschichte. Berlin \& New York: De Gruyter.

Tomasetti, Cristian/Vogelstein, Bert (2015): „Cancer Etiology. Variation in Cancer Risk among Tissues Can Be Explained by the Number of Stem Cell Divisions“. In: Science 347(6217), S. 78-81. 
Tracy, Theodore James (1969): Physiological Theory and the Doctrine of the Mean in Plato and Aristotle. Den Haag: Mouton.

van der Eijk, Philip (2000): „Aristotle’s Psycho-physiological Account of the Soul-Body Relationship“. In: John P. Wright/Paul Potter (Hrsg.): Psyche and Soma. Physicians and Metaphysicians on the Mind-Body Problem. Oxford: Oxford University Press, S. 57-77. van der Eijk, Philip (2005): Medicine and Philosophy in Classical Antiquity. Doctors and Philosophers on Nature, Soul, Health and Disease. Cambridge: Cambridge University Press.

van der Eijk, Philip (2011): „Gesundheit: Eigenverantwortung oder Schicksal?“. In: Deutsches Ärzteblatt International 108(44), S. 2330-2332.

van der Eijk, Philip (2013): „Gesundheit von Leib und Seele in der Antike und im Christentum“. In: Auenzeitung 18(Februar/März), S. 12-13.

van der Eijk, Philip (2016): „On ,Hippocratic‘ and ,Non-Hippocratic“ Medical Writings“. In: Lesley Dean-Jones/Ralph M. Rosen (Hrsg.): Ancient Concepts of the Hippocratic. Leiden \& Boston: Brill, S. $17-47$.

van der Eijk, Philip (2021): „Health, Lifestyle and Responsibility in Greek and Roman Medical and Philosophical Thought“. In: Museum Sinicum 3, S. 159-179.

Wöhrle, Georg (1990): Studien zur Theorie der antiken Gesundheitslehre. Stuttgart: Franz Steiner.

Wu, Song/Powers, Scott/Zhu, Wei et al. (2015): „Substantial Contribution of Extrinsic Risk Factors to Cancer Development“. In: Nature 529(7584), S. 43-47. 